\title{
Zeros of Polynomials in Several Variables and Fractional Order Differences of Their Coefficients
}

\section{B. Mond.* and O. Shisha*}

(May 13, 1964)

\begin{abstract}
A classical result of Eneström (if $c_{0}, c_{1}, \ldots, c_{n}(n \geqslant 1)$ are not all zero and if they satisfy $c_{0} \geqslant c_{1} \ldots$ $\geqslant c_{n} \geqslant 0$, then every zero $\zeta$ of $\sum_{k=0}^{n} c_{k} z^{k}$ satisfies $|\zeta| \geqslant 1$ ) is generalized to polynomials in several variables. A result in the same direction, involving fractional order differences of coefficients, is then established.
\end{abstract}

\section{A Generalization of Eneström's Theorem}

We begin with the following

THEOREM 1. Consider a polynomial $E\left(z_{1}, z_{2}, \ldots, z_{p}\right) \equiv \sum_{h_{p}=0}^{n_{p}} \ldots \sum_{h_{1}=0}^{n_{1}} c_{h_{1}} \ldots h_{p} z_{1}^{h_{1}} \ldots . z_{p}^{h} p(\not \equiv 0)$ in the complex variables $z_{1}, \ldots, z_{p}$. Set $c_{h_{1}} \ldots h_{p}=0$ whenever the $h_{\nu}$ are integers but some $h_{j}$ does not satisfy $0 \leqslant h_{j} \leqslant n_{j}{ }^{1} \quad$ Suppose

$$
\nabla c_{h_{1}} \ldots h_{p}=\sum_{j=1}^{p} c_{h_{1} \ldots h_{p}}-c_{h_{1} \ldots h_{j-1} h_{j}-1 h_{j+1} \ldots h_{p}}=p c_{h_{1}} \ldots h_{p}-\sum_{j=1}^{p} c_{h_{1}} \ldots h_{j-1} h_{j}-1 h_{j+1} \ldots h_{p} \leqslant 0
$$

$\left(h_{\nu}=0,1, \ldots, n_{\nu}+1 ; \nu=1,2, \ldots, p ;\left(h_{1} \ldots h_{p}\right) \neq(0, \ldots 0)\right) . \quad$ If $E\left(\zeta_{1}, \ldots, \zeta_{p}\right)=0$, then at least one $\left|\zeta_{j}\right|$ is $\geqslant 1 .^{2}$

Proof. A straightforward computation yields

$\left(p-\sum_{j=1}^{p} z_{j}\right) E\left(z_{1}, \ldots, z_{p}\right) \equiv \sum_{h_{p}=0}^{n_{p}+1} \ldots \sum_{h_{1}=0}^{n_{1}+1}\left(p c_{h_{1}} \ldots h_{p}-c_{h_{1}-1 h_{2}} \ldots h_{p}-\ldots\right.$

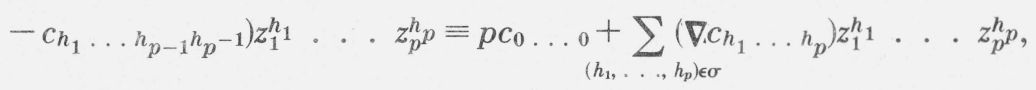

where $\sigma$ is the set of all sequences of integers $\left(h_{1}, \ldots, h_{p}\right)$ with $0 \leqslant h_{\nu} \leqslant n_{\nu}+1, \nu=1,2, \ldots, p$, $\left(h_{1}, \ldots h_{p}\right) \neq(0, \ldots 0)$. Setting $z_{1}=z_{2}=\ldots=z_{p}=1$, we have $p c_{0} \ldots 0_{0}=-\sum\left(\nabla c_{h_{1}} \ldots h_{p}\right)$, and thus $\left.\left(p-\sum_{j=1}^{p} z_{j}\right) E\left(z_{1}, \ldots, z_{p}\right) \equiv \sum_{\left(h_{1}, \ldots, h_{p}\right) \epsilon \sigma}\left(\nabla c_{h_{1}} \ldots h_{p}\right) z_{1}^{h_{1}} \ldots z_{p}^{h_{p}}-1\right)$, from which we infer that $\nabla c_{h_{1}} \ldots h_{p}$ $\neq 0$ for at least one $\left(h_{1} \ldots h_{p}\right) \epsilon \sigma$. If $\left|z_{\nu}\right|<1$ for $\nu=1,2, \ldots, p$ then

*Aerospace Research Laboratories, Wright-Patterson Air Force Base, Ohio.

${ }^{1}$ Below, instead of repeating the last sentence, we shall merely place the sign * at the appropriate place.

${ }^{2}$ Thus, if, for example, $p=2$, the curve $E(x, y)=0$ in the $x, y$ plane does not intersect the square $-1<x<1,-1<y<1$. 
$\operatorname{Re}\left[\left(p-\sum_{j=1}^{p} z_{j}\right) E\left(z_{1}, \ldots, z_{p}\right)\right]=\sum_{\left(h_{1}, \ldots, h_{p}\right) \in \sigma}\left(\nabla c_{h_{1}} \ldots h_{p}\right) \operatorname{Re}\left(z_{1}^{h_{1}} \ldots z_{p}^{h_{p}}-1\right)>0$, and so $E\left(z_{1}, \ldots\right.$ $\left.z_{p}\right) \neq 0$.

When $p=1$, Theorem 1 reduces to a result of Eneström $[1]^{3}$ stating that if $c_{0}, c_{1}, \ldots ., c_{n}(n \geqslant 1)$ are not all zero and if they satisfy $c_{0} \geqslant c_{1} \ldots \geqslant c_{n} \geqslant 0$, then every zero $\zeta$ of $\sum_{k=0}^{n} c_{k} z^{k}$ satisfies $|\zeta| \geqslant 1$.

\section{Fractional Order Differences}

The main purpose of this paper is to generalize a result of Cargo and Sinisha [2, Theorem 1 and the end of IV] relating the zeros of a polynomial (in one variable) to fractional order differences of its coefficients.

Consider a multisequence $a_{h_{1}} \ldots h_{p}\left(p \geqslant 1\right.$ fixed, $\left.h_{\nu}=\ldots-2,-1,0,1,2, \ldots ; \nu=1,2, \ldots, p\right)$. Let $\mathbf{I} a_{h_{1} \ldots h_{p}} \equiv a_{h_{1}} \ldots h_{p}$, and for $j=1,2, \ldots, p$, let $\mathbf{E}_{j} a_{h_{1}} \ldots h_{p} \equiv a_{h_{1}} \ldots h_{j-1} h_{j+1} h_{j+1} \ldots h_{p}$. Let $\alpha$ be a complex number. From (1), one is led to the following symbolic equalities

$$
\begin{aligned}
\nabla^{\alpha}=\left(p \mathbf{I}-\sum_{j=1}^{p} \mathbf{E}_{j}^{-1}\right)^{\alpha}=p^{\alpha} & \left(\mathbf{I}-p^{-1} \sum_{j=1}^{p} \mathbf{E}_{j}^{-1}\right)^{\alpha}=p^{\alpha} \sum_{m=0}^{\infty}(-1)^{m}\left(\begin{array}{c}
\alpha \\
m
\end{array}\right) p^{-m}\left(\sum_{j=1}^{p} \mathbf{E}_{j}^{-1}\right)^{m} \\
& =\sum_{\substack { m=0 \\
\begin{subarray}{c}{i \\
i{ m = 0 \\
\begin{subarray} { c } { i \\
i } }\end{subarray}} \sum_{\substack{k \\
i_{k}=m}}(-1)^{m} m !\left(\begin{array}{c}
\alpha \\
m
\end{array}\right) p^{\alpha-m}\left[i_{1} ! \ldots i_{p} !\right]^{-1} \mathbf{E}_{1}^{-i_{1}} \ldots \mathbf{E}_{p}^{-i_{p}} .
\end{aligned}
$$

Thus, we define

$$
\nabla^{\alpha} a_{h_{1}} \ldots h_{p}=\sum_{m=0}^{\infty} \sum_{\substack{i_{k} \geqslant 0 \\
\Sigma i_{k}=m}}(-1)^{m} m !\left(\begin{array}{l}
\alpha \\
m
\end{array}\right) p^{\alpha-m}\left[i_{1} ! \ldots i_{p} !\right]^{-1} a_{h_{1}-i_{1}} \ldots h_{p}-i_{p}
$$

for every $\left(h_{1}, \ldots, h_{p}\right)$ for which the last infinite sum converges. If $a_{\nu_{1}} \ldots \nu_{p}=0$ whenever some $\nu_{j}$ is $<0$, and if $h_{j} \geqslant 0, j=1,2, \ldots, p$, then

$$
\nabla^{\alpha} a_{h_{1}} \ldots h_{p}=\sum_{m=0}^{h_{1}+\ldots} \sum_{\substack{i_{k} \geqslant 0 \\
\Sigma i_{k}=m}}(-1)^{m} m !\left(\begin{array}{c}
\alpha \\
m
\end{array}\right) p^{\alpha-m}\left[i_{1} ! \ldots i_{p} !\right]^{-1} a_{h_{1}-i_{1}} \ldots h_{p}-i_{p} .
$$

Theorem 2. Consider a polynomial $E\left(z_{1}, \ldots, z_{p}\right) \equiv \sum_{h_{p}=0}^{n_{p}} \ldots \sum_{h_{1}=0}^{n_{1}} c_{h_{1}} \ldots h_{p} z_{1}^{h_{1}} \ldots z_{p}^{h_{p}} \neq 0$ ) in the complex variables $z_{1}, \ldots ., z_{p} .^{*}$ Let $0<\alpha<1$, and suppose that $c_{h_{1}} \ldots h_{p} \geqslant 0\left(h_{\nu}=0\right.$, $\left.1, \ldots ., n_{\nu} ; \nu^{\prime}=1,2, \ldots, p\right), \nabla^{\alpha} c_{h_{1}} \ldots h_{p} \leqslant 0\left(h_{\nu}=0,1, \ldots . n_{\nu} ; \nu=1,2, \ldots, p ;\left(h_{1}, \ldots ., h_{p}\right)\right.$ $\neq(0, \ldots, 0)) . \quad$ If $E\left(\zeta_{1}, \ldots, \zeta_{p}\right)=0$, then at least one $\left|\zeta_{j}\right|$ is $>1$.

Proof. For $\left|\sum_{j=1}^{p} z_{j}\right| \leqslant p$ we have ${ }^{4}$

$$
\begin{aligned}
& \left(1-p^{-1} \sum_{j=1}^{p} z_{j}\right)^{\alpha} E\left(z_{1}, \ldots, z_{p}\right)=\left[\sum_{j=0}^{\infty}\left(\begin{array}{c}
\alpha \\
j
\end{array}\right)(-1)^{j} p^{-j}\left(\sum_{\nu=1}^{p} z_{\nu}\right)^{j}\right] \sum_{j=0}^{\infty} \sum_{\substack{h_{k} \geqslant 0 \\
\Sigma h_{k}=j}} c_{h_{1}} \ldots h_{p} z_{1}^{h_{1}} \ldots z_{p}^{h_{p} p} \\
& =\sum_{j=0}^{\infty} \sum_{m=0}^{j}\left\{\sum_{\substack{i_{k} \geqslant 0 \\
\Sigma i_{k}=m}}(-1)^{m} m !\left(\begin{array}{l}
\alpha \\
m
\end{array}\right) p^{-m}\left[i_{1} ! \ldots i_{p} !\right]^{-1} z_{1}^{i_{1}} \ldots z_{p}^{i p}\right\}\left\{\sum_{\substack{h_{k} \geqslant 0 \\
\Sigma h_{k}=j-m}} c_{h_{1}} \ldots h_{p} z_{1}^{h_{1}} \ldots z_{p}^{h_{p}}\right\}
\end{aligned}
$$

\footnotetext{
${ }^{3}$ Figures in brackets indicate the literature references at the end of this paper.
}

${ }^{4} \mathrm{By} z^{\alpha}$, for a complex $z$, we mean the principal value of that power $(0$ if $z=0)$. 


$$
\begin{aligned}
& =\sum_{j=0}^{\infty} \sum_{m=0}^{j} \sum_{\substack{i_{k} \geqslant 0 \\
\Sigma i_{k}=m}}(-1)^{m} m !\left(\begin{array}{l}
\alpha \\
m
\end{array}\right) p^{-m}\left[i_{1} ! \ldots i_{p} !\right]^{-1} c_{q_{1}-i_{1}} \ldots q_{p}-i_{p} z_{1}^{q_{1}} \ldots z_{p}^{q_{p}} \\
& q_{k} \geqslant i k \text {. } \\
& \Sigma q_{k}=j
\end{aligned}
$$

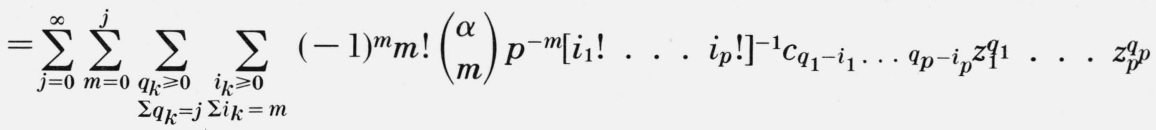

$$
\begin{aligned}
& =\sum_{j=0}^{\infty} \sum_{\substack{q_{k} \geqslant 0 \\
\Sigma q_{k}=j}} \sum_{\substack{m=0 \\
\Sigma i_{i}=m}}^{\infty} \sum_{\substack{i_{k} \geqslant 0 \\
\Sigma(1)^{2}}}(-1)^{m} m !\left(\begin{array}{c}
\alpha \\
m
\end{array}\right) p^{-m}\left[i_{1} ! \ldots i_{p} !\right]^{-1} c_{q_{1}-i_{1}} \ldots q_{p}-i_{p} z_{1}^{q_{1}} \ldots z_{p}^{q_{p}} \\
& =\sum_{j=0}^{\infty} \sum_{\substack{q_{k} \geqslant 0 \\
\Sigma \\
q_{k}=j}} p^{-\alpha}\left(\nabla^{\alpha} c_{q_{1}} \ldots q_{p}\right) z_{1}^{q_{1}} \ldots z_{p}^{q_{p}} .
\end{aligned}
$$

Setting $z_{1}=\ldots=z_{p}=1$, we get $0=p^{-\alpha} \nabla^{\alpha} c_{0} \ldots 0+\sum_{j=1}^{\infty} \sum_{\substack{q_{k} \geqslant 0 \\ \Sigma q_{k}=j}} p^{-\alpha} \nabla^{\mid \alpha} c_{q_{1}} \ldots q p$;

Thus, for $\left|\sum_{j=1}^{p} z_{j}\right| \leqslant p$ we have

$$
\left(p-\sum_{j=1}^{p} z_{j}\right)^{\alpha} E\left(z_{1}, \ldots, z_{p}\right)=\sum_{j=1}^{\infty} \sum_{\substack{q_{k} \geqslant 0 \\ \Sigma q_{k}=j}}\left(\nabla^{a} c_{q_{1}} \ldots q_{p}\right)\left(z_{1}^{q_{1}} \ldots . z_{p}^{q_{p}}-1\right) .
$$

Let $q_{1}, \ldots, q_{p}$ be arbitrary integers $\geqslant 0$ (not all zero). Then $\nabla^{\alpha} c_{q_{1}} \ldots q_{p} \leqslant 0$. To see this we may assume that some $q_{j}$ is $>m_{j}$. But then, noting that $(-1)^{m}\left(\begin{array}{c}\alpha \\ m\end{array}\right)<0$ for $m=1,2, \ldots$, we have

$$
\nabla^{\alpha} c_{q_{1}} \ldots q_{p}=\sum_{m=1}^{q_{1}+\ldots+q_{p}} \sum_{\substack{i_{k} \geqslant 0 \\
\Sigma i_{k}=m}}(-1)^{m} m !\left(\begin{array}{c}
\alpha \\
m
\end{array}\right) p^{\alpha-m}\left[i_{1} ! \ldots i_{p} !\right]^{-1} c_{q_{1}-i_{1}} \ldots q_{p}-i_{p} \leqslant 0 .
$$

From (2) we obtain, for $\left|\sum_{j=1}^{p} z_{j}\right| \leqslant p$

$$
\operatorname{Re}\left\{\left(p-\sum_{j=1}^{p} z_{j}\right)^{\alpha} E\left(z_{1}, \ldots, z_{p}\right)\right\}=\sum_{j=1}^{\infty} \sum_{\substack{q_{k} \geqslant 0 \\ \Sigma q_{k}=j}}\left(\nabla^{\alpha} c_{q_{1}} \ldots q_{p}\right) \operatorname{Re}\left(z_{1}^{q_{1}} \ldots . z_{p}^{\left.q_{p}-1\right) .}\right.
$$

Suppose $E\left(\zeta_{1}, \ldots, \zeta_{p}\right)=0$, where all $\left|\zeta_{j}\right|$ are $\leqslant 1$. If $q_{1}, \ldots, q_{p}$ are non-negative integers (not all zero), then $\operatorname{Re}\left(\zeta_{1}^{q_{1}} \ldots \zeta_{p}^{q} p-1\right) \leqslant 0$, and equality must hold if $\nabla^{\alpha} c_{q_{1}} \ldots q_{p}<0$. Let $j(1 \leqslant j \leqslant p)$ be an integer. Since some $c_{h_{1}} \ldots h_{p}$ must be positive, we have

$$
\begin{aligned}
& \nabla^{\alpha} c_{n_{1}} \ldots n_{j-1} n_{j+1} n_{j+1} \ldots n_{p} \\
& \quad{ }_{m}^{n_{1}+\ldots+n_{p}+1} \sum_{\substack{i_{k} \geq 0 \\
\Sigma i_{k}=m}}(-1)^{m} m !\left(\begin{array}{l}
\alpha \\
m
\end{array}\right) p^{\alpha-m}\left[i_{1} ! \ldots i_{p} !\right]^{-1} c_{n_{1}}-i_{1} \ldots n_{j-1}-i_{j-1} n_{j}+1-i_{j} n_{j+1}-i_{j+1} \ldots n_{p}-i_{p}<0
\end{aligned}
$$

and similarly $\nabla^{\alpha} c_{n_{1}} \ldots n_{j-1} n_{j+2 n_{j+1}} \ldots n_{p}<0$. Thus 
$\operatorname{Re}\left(\zeta_{1}^{n_{1}} \ldots \zeta_{j-1}^{n_{j-1}} \zeta_{j}^{n_{j+1}} \zeta_{j+1}^{n_{j+1}} \ldots \zeta_{p}^{n_{p}}-1\right)=\operatorname{Re}\left(\zeta_{1}^{n_{1}} \ldots \zeta_{j-1}^{n_{j-1}} \zeta_{j}^{n_{j+2}} \zeta_{j+1}^{n_{j+1}} \ldots . \zeta_{p}^{n_{p}}-1\right)=0$, and so

$$
\zeta_{1}^{n_{1}} \ldots \zeta_{j-1}^{n_{j-1}} \zeta_{j}^{n_{j+1}} \zeta_{j+1}^{n_{j+1}} \ldots \zeta_{p}^{n_{p}}=1=\zeta_{1}^{n_{1}} \ldots \zeta_{j-1}^{n_{j-1}} \zeta_{j}^{n_{j+2}} \zeta_{j+1}^{n_{j+1}} \ldots \zeta_{p}^{n_{p}}
$$

which implies that $\zeta_{j}=1$. Thus $\zeta_{1}=\zeta_{2}=\ldots=\zeta_{p}=1$, and so $0=E(1,1, \ldots, 1)$

$=\sum_{h_{p}=0}^{n_{p}} \cdots \sum_{h_{1}=0}^{n_{1}} c_{h_{1}} \ldots h_{p}>0$, which proves the Theorem.

\section{Remarks} We consider a polynomial $E\left(z_{1}, \ldots, z_{p}\right) \equiv \sum_{h_{p}=0}^{n_{p}} \ldots \sum_{h_{1}=0}^{n_{1}} \alpha_{h_{1}} \ldots h_{p} z_{1}^{h_{1}} \ldots z_{p}^{h_{p}}(\not \equiv 0)$ in the
complex variables $z_{1}, \ldots, z_{p}$.

A. Suppose the $\alpha_{h_{1}} \ldots h_{p}$ are complex. Let $r_{1}, \ldots, r_{p}$ be complex, non-zero numbers, and set $c_{h_{1}} \ldots h_{p}=\alpha_{h_{1}} \ldots h_{p} r_{1}^{h_{1}} \ldots r_{p}^{h}\left(h_{\nu}=0,1, \ldots, n_{\nu} ; \nu=1,2, \ldots, p\right){ }^{*} \quad$ Assume $\nabla c_{h_{1}} \ldots h_{p} \leqslant 0$ $\left(h_{\nu}=0,1, \ldots, \dot{n}_{\nu}+1 ; \nu=1,2, \ldots, p ;\left(h_{1}, \ldots, h_{p}\right) \neq(0, \ldots 0)\right)$. Suppose $E\left(\zeta_{1}, \ldots, \zeta_{p}\right)=0$. Then at least one $\left|\zeta_{j}\right|$ is $\geqslant\left|r_{j}\right|$. Indeed,

$$
E\left(z_{1}, \ldots, z_{p}\right) \equiv \sum_{h_{p}=0}^{n_{p}} \ldots \sum_{h_{1}=0}^{n_{1}} c_{h_{1}} \ldots h_{p}\left(\frac{z_{1}}{r_{1}}\right)^{h_{1}} \ldots\left(\frac{z_{p}}{r_{p}}\right)^{h_{p}}
$$

and therefore by Theorem 1, at least one $\left|\zeta_{j} / r_{j}\right|$ is $\geqslant 1$.

As a simple example, take $p=1, n_{1}=n(\geqslant 1)$, and assume the $\alpha_{\nu}$ are alternating in sign: $\alpha_{\nu}=(-1)^{\nu}\left|\alpha_{\nu}\right|(\nu=0,1, \ldots, n)$ and $\left|\alpha_{0}\right| \geqslant\left|\alpha_{1}\right| \ldots . \geqslant\left|\alpha_{n}\right|$.

By taking $r_{1}=-1$, we obtain that every zero of the polynomial $\sum_{\nu=0}^{n} \alpha_{\nu} z^{\nu}$ has a modulus $\geqslant 1$. B. Suppose that $E\left(z_{1}, \ldots, z_{p}\right)$ is not a constant and that the $\alpha_{h_{1}} \ldots h_{p}$ are $\geqslant 0$. Let

$$
r=\min \left[\left(\sum_{\substack{j=1 \\ h_{j} \geqslant 1}}^{p} \alpha_{h_{1}} \ldots h_{j-1} h_{j}-1 h_{j+1} \ldots h_{p}\right) / p \alpha_{h_{1}} \ldots h_{p}\right]
$$

where the minimum is taken over all sequences $\left(h_{1}, \ldots, h_{p}\right)$ with $0 \leqslant h_{\nu} \leqslant n_{\nu}(\nu=1,2, \ldots, p)$, $\left(h_{1}, \ldots, h_{p}\right) \neq(0, \ldots, 0), \alpha_{h_{1}} \ldots h_{p} \neq 0$. Suppose $E\left(\zeta_{1}, \ldots, \zeta_{p}\right)=0$. Then at least one $\left|\zeta_{j}\right|$ is $\geqslant r$.

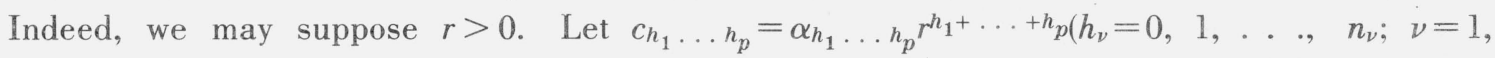
$2, \ldots, p)$.* $^{*} \quad$ Let $0 \leqslant h_{\nu} \leqslant n_{\nu}+1, \nu=1,2, \ldots, p ;\left(h_{1}, \ldots . h_{p}\right) \neq(0, \ldots, 0)$. We shall show that $\nabla c_{h_{1}} \ldots h_{p} \leqslant 0$. We may clearly assume that all $h_{\nu}$ are $\leqslant n_{\nu}$ and that $\alpha_{h_{1}} \ldots h_{p} \neq 0$. Then $\nabla c_{h_{1}} \ldots h_{p}=r^{h_{1}+\ldots+h_{p}-1}\left[r p \alpha_{h_{1}} \ldots h_{p}-\sum_{\substack{j=1 \\ h_{j} \geqslant 1}}^{p} \alpha_{h_{1}} \ldots h_{j-1} h_{j}-1 h_{j+1} \ldots h_{p}\right] \leqslant 0 . \quad$ By A, at least
one $\left|\zeta_{j}\right|$ is $\geqslant r$.

\section{References}

[1] Eneström, G., Härledning af en allmän formel för antalet pensionärer, som vid en godtycklig tidpunkt förefinnas inom en sluten pensionskassa. Öfversigt af Kongl. Vetenskaps Akad. Förhandl. 50, 405-415 (1893).

[2] Cargo, G. T., and Shisha, O., Zeros of polynomials and fractional order differences of their coefficients. J. Math. Analysis and Applications 7, 176-182 (1963).

(Paper 68B3-124) 